(C) [2009] IEEE. Reprinted, with permission, from [Sithamparanathan Kandeepan, Sam Reisenfeld, Tuncer Can Aysal, David Lowe, Radoslaw Piesiewicz, Bayesian Tracking in Cooperative Localization for Cognitive Radio Networks, Vehicular Technology Conference, 2009. VTC Spring 2009. IEEE 69th]. This material is posted here with permission of the IEEE. Such permission of the IEEE does not in any way imply IEEE endorsement of any of the University of Technology, Sydney's products or services. Internal or personal use of this material is permitted. However, permission to reprint/republish this material for advertising or promotional purposes or for creating new collective works for resale or redistribution must be obtained from the IEEE by writing to pubs-permissions@ieee.org. By choosing to view this document, you agree to all provisions of the copyright laws protecting it 


\title{
Bayesian Tracking in Cooperative Localization for Cognitive Radio Networks
}

\author{
$\dagger$ Sithamparanathan Kandeepan, § Sam Reisenfeld, ` Tuncer Can Aysal, § David Lowe, ${ }^{\dagger}$ Radoslaw Piesiewicz, \\ $\dagger$ Broadband and Wireless Lab, Create-Net International Research Centre, \\ via alla Cascatta 56D, Trento, Centre for Italy, \\ $\S$ Centre for Real-Time Information Networks (CRiN), Faculty of Engineering, University Technology, Sydney \\ 1 Broadway, NSW 2007, Australia \\ Email: kandeepan@ieee.org,samr@uts.edu.au,tuncer.aysal@create-net.org, \\ david.lowe@uts.edu.au,radoslaw.piesiewicz@create-net.org,
}

\begin{abstract}
In this paper we present some work on cooperative localization and tracking of primary users (PU) in a cognitive radio network using Bayesian techniques. We use particle filtering methods to track the location of a PU in the network using cooperative localization techniques and present some results for noisy measurements. The cognitive radio $(\mathrm{CR})$ nodes estimate the information related to the geographical position of the PU based on existing location identification and localization techniques and forward the noisy information to a cognitive radio base station (CRB), which then fuses the information to estimate the position of the PU in the network in order to perform a radio scene analysis. We propose a particle filtering approach that is suitable for tracking non-Gaussian noisy signals at the CRB to estimate the position of a $\mathrm{PU}$, two importance-functions relative to the particle filtering algorithm are also presented. Simulations are performed on the proposed tracking algorithm and the results are presented in terms of the mean square error of the positional estimates.
\end{abstract}

\section{INTRODUCTION}

There has been a shift in the trend on wireless communications recently with the introduction of Cognitive Radios (CR) and related concepts [1] - [4]. CR networks are known to share the spectrum in an opportunistic manner with the other co-existing radios by considering them as the primary users (PU). This is considered to be a better way of efficiently utilizing the electro-magnetic radio spectrum. The concept has especially encouraged and motivated the spectrum policy regulators around the world [5], [6] to investigate into the technology further. For such technology to be feasible, the $\mathrm{CR}$ nodes need to be aware of the surrounding users of the radio spectrum (i.e. the PUs) within the region of interest, hence $\mathrm{CR}$ networks require the intelligence of tracking and localizing the PU present within the vicinity of the network to avoid interfering with their transmissions. This motivates us to study precise localization and tracking techniques for CR networks. In this paper, we investigate the performance of a cooperative tracking system to track PUs based on the Bayesian principles [8], [7] for CR networks. High precision tracking is required especially for indoor environments (home theater environments) where the target needs to be localized within a few tens of centimetres, therefore we consider Bayesian estimation technique to be a suitable candidate.

Here we present a summary of the existing localization methods in the literature which are mainly focused on wireless sensor networks, and we clearly see that such techniques are directly applicable for CR networks as well. As mentioned above, one of the important tasks that a CR network needs to perform is target location estimation, which is imperative for an accurate tracking of the target and higher level of motion analysis. Many methods for acoustic source localization in sensor networks or sensor arrays are available in the literature [9], [10], [11], [12], [13]. Techniques based on Direction of Arrival (DOA) have been investigated for narrowband sources [9], [10], [11]. For broadband sources, methods based on TimeDelay of Arrival (TDOA) estimation are more suitable [12], [13]. In a CR network, the CR nodes are usually not precisely synchronized, and hence, the TDOA, which requires accurate timing of the signals, is not a good practical solution. In such a network, typically there can be a large number of CR nodes (with respect to the size of the region) which are densely deployed in the given Region of Interest (ROI), which then makes accurate intensity (energy) based target localization possible [14], [15], [16], [17], [18], [19]. Signal intensity measurements are usually used for target detection, hence, it is very convenient and economical to utilize them to localize a target, without the need for additional sensor functionalities and measurement features, such as DOA [14], [9], [10], [11]. Energy based methods, hence, have been proposed and developed such as in [14], [15], [16], [17], [18], [19]. Least squares methods are proposed to localize a single acoustic source based on energy ratios [17]. A maximum likelihood (ML) based acoustic source localization method has been presented [16]. The source localization is also considered in a distributed manner, without transmitting the full data set to a central processing point [15], [18], [19].

In our work presented in this paper, the CR nodes perform a noisy measurement on the location of the PU by means of an existing technique, and the corresponding location estimation information are transmitted to the CR base-station (CRB). The 
CRB then fuses the received information and keeps track of the particular PU with a higher precision of accuracy. We present a model to perform cooperative tracking of PUs in a CR network using particle filtering techniques [21], [22] in order to obtain higher precision. One of the main reasons to choose particle filters for tracking is due to the existence of non-Gaussian additive noise in the estimation of the phase angle which is used localize the source.

In Section-II, we describe the location assisted wireless systems for CR networks, and in Section-III we present the cooperative localization and tracking model proposed for CR networks. In Section-IV we present the particle filtering algorithm to track the PU and in Section-V we perform some simulations and show some results on the performance of the tracking scheme. Finally, in Section-VI we provide some concluding remarks.

\section{LOCATION ASSisted WiRELESS SyStems}

Location assisted systems are increasingly getting popular within cognitive radio networks for dynamic spectrum management. In a cognitive radio network for cooperative communications the cognitive radio base station (CRB) creates a radio map of the environment within the vicinity of the network by identifying the location of the radios or the PUs. Creating such a radio environment map is a convenient way of performing dynamic spectrum management within the network and also assisting $\mathrm{CR}$ nodes with its radio transmissions especially for UWB based nodes such that the PU are not interfered with the CR transmissions. The FCC has adopted this method as one of the techniques for dynamic spectrum management in radio networks. In an an FCC proposed method, a geolocation database is created at a central node by identifying the primary users that are equipped with location identification devices which inform their location to the central database frequently. The UWB based CR networks however locates PUs by performing location estimation based on statistical signal information and combining them to form a radio environment map similar to the geolocation database. The radio environment map which contains the space-frequency information of the PUs is then used by the CR network to reuse the spectrum efficiently. The CRB distributes this information to the CRs in order to control the transmission parameters such as the power levels, frequency bands and the direction of transmissions to avoid interfering with the PU. Figure-1 shows a typical radio environment map created by a CRB in a CR network. A typical radio environment map however contains several other information other than the ones that are of interest to us here such as the frequency, power and space information. Some of the other information that could be possibly contained within the radio environment map are service and network information, regulatory information and radio device information etc. In the figure, two distinctive advantages are depicted in terms of interference reduction and radio transmission using the knowledge of the radio environment map. The first advantage is, the $\mathrm{CR}$ nodes can perform directional transmissions by knowing the location of

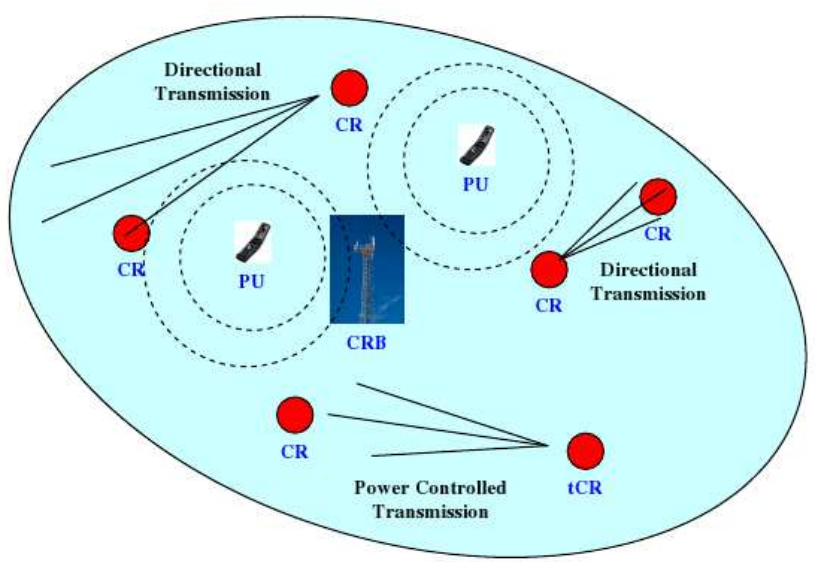

Fig. 1. A typical radio environment map and radio scene analysis in a cognitive radio network environment: $\mathrm{CR}$ - cognitive radio, CRB - cognitive radio base station

the PUs in the surroundings and avoid potential interferences to them, the second advantage is that the CR nodes can control the transmission power again to avoid interfering with the PU in the surrounding environment.

\section{CoOperative Localization Model}

In this section we present the cooperative localization model and strategy that is considered in this paper. The CR nodes in the network perform individual estimates of the time of arrival (ToA) and the direction of arrival (DoA) on the nearest target and pass the information to the CRB. The information conveying link between the $C R$ nodes and the $C R B$ is considered to be an error free channel. We assume that the network is self aware of the positional information of its CR nodes, and that the CRB is the point of origin of the 2D-plane. We formulate the target localization problem as described in [20] for wireless sensor networks. Let us define the set of parameters estimated at the CR nodes as,

$$
\Lambda=\left[R_{i j}(t), \theta_{i j}(t)\right]^{T}
$$

where, $R_{i j}(t)$ is the range computed from the ToA estimates, and $\theta_{i j}(t)$ is the angle of arrival which describes the directional information of the target PU-j at the $i^{t h} \mathrm{CR}$ node, given by,

$$
R_{i j}=\left[\begin{array}{ccccc}
R_{11} & R_{12} & \cdot & \cdot & R_{1 L} \\
R_{21} & R_{22} & \cdot & \cdot & R_{2 L} \\
\cdot & \cdot & \cdot & \cdot & \cdot \\
R_{K 1} & R_{K 2} & \cdot & \cdot & R_{K L}
\end{array}\right]
$$

and,

$$
\theta_{i j}=\left[\begin{array}{ccccc}
\theta_{11} & \theta_{12} & \cdot & \cdot & \theta_{1 L} \\
\theta_{21} & \theta_{22} & \cdot & \cdot & \theta_{2 L} \\
\cdot & \cdot & \cdot & \cdot & \cdot \\
\theta_{K 1} & \theta_{K 2} & \cdot & \cdot & \theta_{K L}
\end{array}\right]
$$




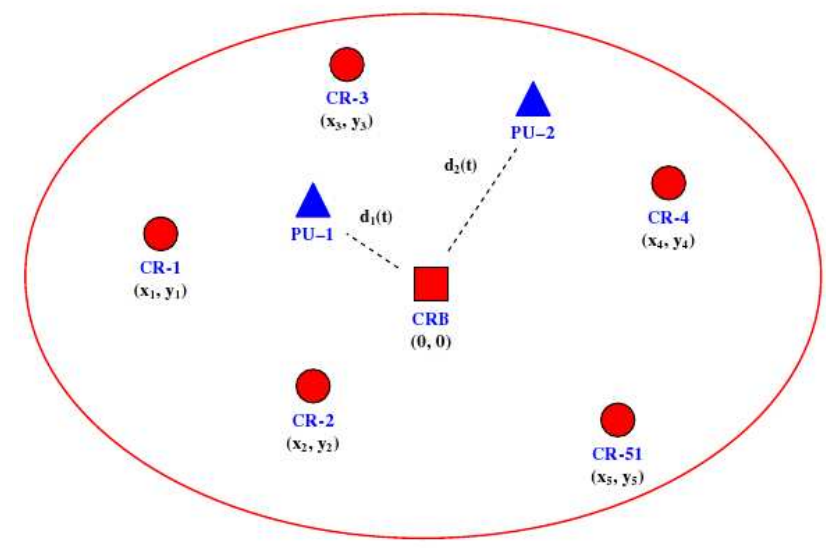

Fig. 2. Cooperative localization of primary users in a cognitive radio network

Then, at the CRB, by using the maximum likelihood (ML) estimation method described in [20], together with the $a$-priori knowledge of the CR node locations, we can arrive at the following noisy estimates for the targets PU-j as,

$$
\begin{gathered}
x_{j}(t)=d_{j}(\Lambda)+w_{d}^{j}(t) \\
\Phi_{j}(t)=\phi_{j}(\Lambda)+w_{\phi}^{j}(t)
\end{gathered}
$$

In (4) and (5), $d_{j}(\Lambda)$ and $\phi_{j}(\Lambda)$ are the true values of the distances and the phase angles of the PU-j's with respect to the CRB in the absence of the observation noise, and $w_{d}^{j}(t)$ and $w_{\phi}^{j}(t)$ are the additive noise components corresponding to the ML estimations. The signal to noise ratio of the received signals prior to the ML estimation are given by $\rho_{j}$ and $\varsigma_{j}$ for the range and the phase respectively. The additive noise component $w_{d}^{j}(t)$ is modeled as a Gaussian random process with a zero mean and a variance of $\sigma_{d_{j}}^{2}$, and $w_{\phi}^{j}(t)$ is modeled as a zero mean random process with a phase noise distribution given by equation (6) [23], [24]. The mobility of the PUs is modeled by two first order Markov processes for the distance and the phase angle described by the density functions $p\left(d_{j}^{t} \mid d_{j}^{t-1}\right)$ and $p\left(\phi_{j}^{t} \mid \phi_{j}^{t-1}\right)$, with $d_{j}^{t}$ and $\phi_{j}^{t}$ being the distance and the angle at time $t$.

The positional estimates $x_{j}$ and $\Phi_{j}$ are then used for tracking the target PU-j in the network. In the following section we look at a Bayesian method for continuous tracking of the target based on the estimates from (4) and (5).

\section{BAYESIAN TRACKING OF PRIMARY USERS}

Bayesian estimation and tracking is a well studied topic when the posteriori distribution of the noise process is known. In this section, by using the cooperative localization model and the estimation noise models described in the previous section, we propose a Bayesian tracking algorithm, namely a particle filtering method, to improve the precision of the location estimation for Gaussian and non-Gaussian noise. Particle filtering is known to be a powerful tracking technique based on sequential Monte-Carlo methodology for sequential signal processing. Consider the discrete model of (4) and (5) given by $x_{j}[k]$ and $\Phi_{j}[k]$ with $k=0,1, \ldots N$. Then, given the observation vector $\mathbf{z}_{\mathbf{k}}=\left[x_{j}[k],\left\{\phi_{j}[k]\right]^{T}\right.$ for the parameter vector $\boldsymbol{\mu}_{\mathbf{k}}=\left[d_{j}[k], \Phi_{j}[k]\right]^{T}$ at the observation time $k$, the Bayesian rule for the iterative estimation of the posteriori pdf $p\left(\boldsymbol{\mu}_{\mathbf{k}} \mid \mathbf{z}_{k}\right)$ is given by,

$$
p\left(\boldsymbol{\mu}_{k} \mid \mathbf{z}_{k}\right)=\frac{p\left(\mathbf{z}_{k} \mid \boldsymbol{\mu}_{\mathbf{k}}\right) p\left(\boldsymbol{\mu}_{\mathbf{k}} \mid \mathbf{z}_{\mathbf{k}-1}\right)}{p\left(\mathbf{z}_{\mathbf{k}} \mid \mathbf{z}_{\mathbf{k}-1}\right)}
$$

where, the denominator is computed from,

$$
p\left(\mathbf{z}_{\mathbf{k}} \mid \mathbf{z}_{\mathbf{k}-\mathbf{1}}\right)=\int p\left(\mathbf{z}_{\mathbf{k}} \mid \boldsymbol{\mu}_{\mathbf{k}}\right) p\left(\boldsymbol{\mu}_{\mathbf{k}} \mid \mathbf{z}_{\mathbf{k}-\mathbf{1}}\right) d \boldsymbol{\mu}_{\mathbf{k}}
$$

Since the elements of $\boldsymbol{\mu}_{\mathbf{k}}$ are first order Markovian processes, the normalizing component of (8) given in (9) can be ignored in the iteration process when computing the posteriori density function. Then, the Bayesian estimate is the expected value of the latest density function $p\left(\boldsymbol{\mu}_{\mathbf{k}} \mid \mathbf{z}_{\mathbf{k}}\right)$, given by,

$$
\hat{\boldsymbol{\mu}}_{\mathbf{k}}=\int \boldsymbol{\mu}_{\mathbf{k}} p\left(\boldsymbol{\mu}_{\mathbf{k}} \mid \mathbf{z}_{\mathbf{k}}\right) d \boldsymbol{\mu}_{\mathbf{k}}
$$

In the next sub section we use particle filtering methods to iteratively estimate $\boldsymbol{\mu}_{\mathbf{k}}$

\section{A. Particle Filtering}

We present a general particle filtering algorithm to track the parameters of interest $d_{j}$ and $\phi_{j}$ based on the model given in the previous section. The probability distributions of interest to us are approximated by considering the discrete version of the distributions, given by,

$$
p(\boldsymbol{\mu} \mid \mathbf{z}) \approx \sum_{n=1}^{M} \boldsymbol{W}_{\mathbf{n}} \delta\left(\boldsymbol{\mu}-\mathbf{u}_{\mathbf{n}}\right)
$$

where, $\mathbf{u}_{\mathbf{n}}=\left[u_{d_{j}}^{n}, u_{\phi_{j}}^{n}\right]^{T}$ are known as the particles that are drawn from the posteriori distribution $p(\boldsymbol{\mu} \mid \mathbf{z}), \boldsymbol{W}_{\mathbf{n}}=$ $\left[w_{d_{j}}^{n} w_{\phi_{j}}^{n}\right]^{T}$ are the corresponding weights assigned to the particles and $M$ is the total number of particles considered. The weights are assigned an initial value based on the particles, typically $w_{d_{j}}^{n}=1 / M, w_{\phi_{j}}^{n}=1 / M$ at $k=0$, and are iteratively computed using (8). The updates of the weights are conveniently computed using the analytical solutions to the posteriori distributions of the parameters of interest. The weights are therefore updated according to,

$$
\begin{gathered}
\bar{w}_{d_{j}}^{n}(k) \propto \bar{w}_{d_{j}}^{n}(k-1) F_{d_{j}}\left(x_{j}(k) \mid d_{j}(k-1)\right) \\
w_{d_{j}}^{n}(k)=\bar{w}_{d_{j}}^{n}(k) / \sum_{n=1}^{M} \bar{w}_{d_{j}}^{n}(k)
\end{gathered}
$$

and,

$$
\begin{gathered}
\bar{w}_{\phi_{j}}^{n}(k) \propto \bar{w}_{\phi_{j}}^{n}(k-1) F_{\phi_{j}}\left(\Phi_{j}(k) \mid \phi_{j}(k-1)\right) \\
w_{\phi_{j}}^{n}(k)=\bar{w}_{\phi_{j}}^{n}(k) / \sum_{n=1}^{M} \bar{w}_{\phi_{j}}^{n}(k)
\end{gathered}
$$




$$
f_{w_{\phi}^{j}}\left(w_{\phi}^{j}\right)= \begin{cases}\exp \left(-\varsigma_{j} / 2\right)\left[\frac{1}{2 \pi}-\exp \left(\frac{\Gamma}{2}\right)\left(\frac{\Gamma}{2 \pi}\right)^{\frac{1}{2}} Q(\sqrt{\Gamma})\right] & \text { for } \pi / 2<w_{\phi}^{j} \leq \pi \\ \exp \left(-\varsigma_{j} / 2\right)\left[\frac{1}{2 \pi}+\exp \left(\frac{\Gamma}{2}\right)\left(\frac{\Gamma}{2 \pi}\right)^{\frac{1}{2}}(1-Q(\sqrt{\Gamma}))\right] & \text { for }-\pi / 2<w_{\phi}^{j} \leq \pi / 2 \\ \exp \left(-\varsigma_{j} / 2\right)\left[\frac{1}{2 \pi}-\exp \left(\frac{\Gamma}{2}\right)\left(\frac{\Gamma}{2 \pi}\right)^{\frac{1}{2}} Q(\sqrt{\Gamma})\right] & \text { for }-\pi<w_{\phi}^{j} \leq-\pi / 2\end{cases}
$$

$$
\text { where, } \Gamma=\varsigma_{j} \cos ^{2}\left(w_{\phi}^{j}\right) \text { and }, Q(z)=\frac{1}{2 \pi} \int_{z}^{\infty} \exp \left(-u^{2} / 2\right) d u
$$

where, $F_{d_{j}}\left(x_{j}(k) \mid d_{j}(k-1)\right)$ and $F_{\phi_{j}}\left(\Phi_{j}(k) \mid \phi_{j}(k-1)\right)$ are known as the importance functions. According to our noise models in (4) and (5), and the corresponding posteriori distributions, we come up with two expressions for the importancefunctions, given by,

$$
\begin{aligned}
& F_{d_{j}}\left(x_{j}(k) \mid d_{j}(k-1)\right)=\exp \left(-\left(u_{d_{j}}^{n}-x_{j}(k)\right)\right) \\
& F_{\phi_{j}}\left(\Phi_{j}(k) \mid \phi_{j}(k-1)\right)=\Upsilon \exp (\Upsilon)(1-Q(\Upsilon))
\end{aligned}
$$

where, $\Upsilon=\cos \left(u_{\phi_{j}}^{n}-\Phi_{j}(k)\right)$. The newly computed particle weights are then used to estimate the parameters by taking the expectation of the discrete sample set,

$$
\hat{d}_{j}(k)=\sum_{n=1}^{M} u_{d_{j}}^{n} w_{d_{j}}^{n} \text { and } \hat{\phi}_{j}(k)=\sum_{n=1}^{M} u_{\phi_{j}}^{n} w_{\phi_{j}}^{n}
$$

After a few iterations (i.e. few estimations), some of the particles will have almost zero weights which are no longer required for the estimation process. In such situations importance sampling [21], [22] is used to reduce the computational burden associated with the estimation process.

\section{Simulation Results}

In this section we present some simulation results for tracking PUs using the particle filtering approach described in the previous section. The simulations were performed assuming the same signal to noise ratio SNR levels for the range and the phase; SNR $=\varsigma_{j}=\rho_{j}$. We also consider a very simple model for the mobility with very slow variation in the position of the PU with time with respect to the CRB. Two separate particle filters are used to track $d_{j}(k)$ and $\phi_{j}(k)$ for every PU. The outputs of the particle filters are then used generate a radio map environment. Figure-3 shows the construction of the posteriori distribution using the particle filtering approach for the phase $\phi_{j}(k)$ with time. The evolution of the distribution is a time depended process as we see from the figure which also depends on SNR.

The corresponding time domain plots for the convergence of the parameters are shown in Figure-4, for various values of SNR and $M=100$. From the figure we observe that the filter takes significant amount of time to converge in terms of the number of samples, but on the other hand shows very low jitter during the steady state. This is the main advantage claimed in our work to perform tracking with greater precision. The jitter performance of the particle filters are depicted in Figure- 5 for

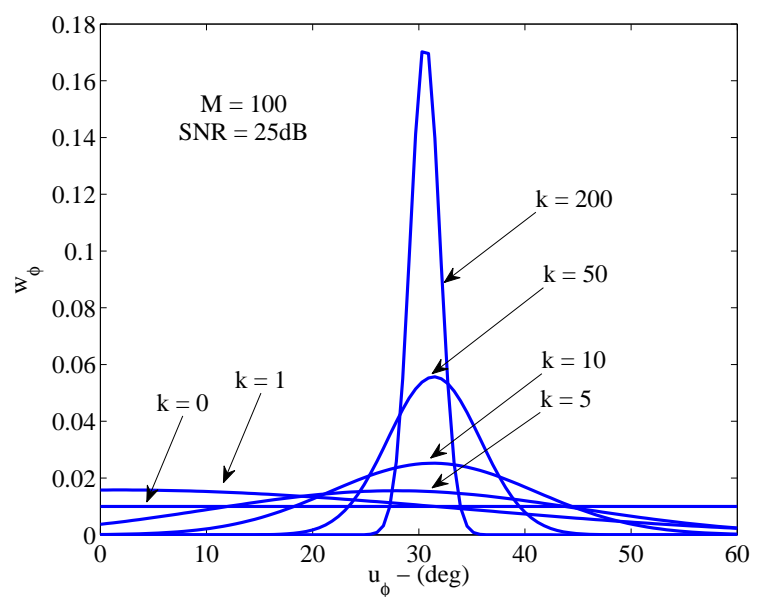

Fig. 3. Construction of the posteriori pdf using the particle filter approach

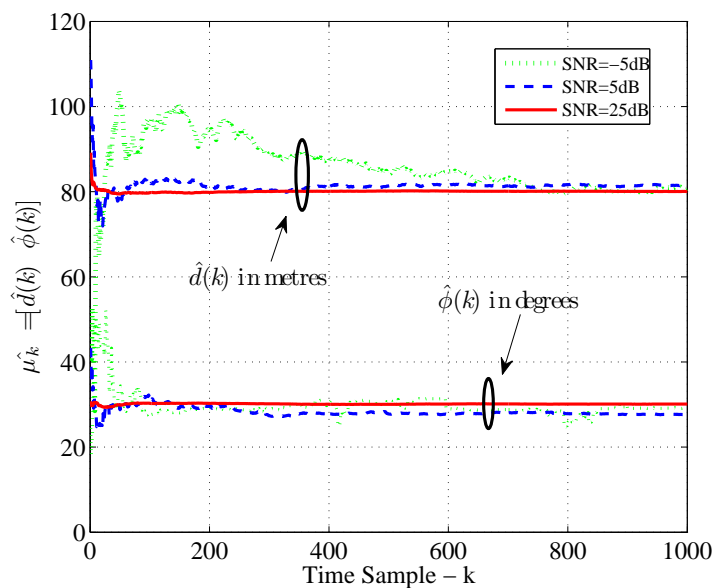

Fig. 4. Bayesian tracking of range $d_{j}(k)$ and phase $\phi_{j}(k), M=100$

various SNR levels. It should be noted that the performance curves depicted here are based on the performances of the ML estimates made prior to the tracking process, and we assume perfect ML estimation for estimating $\Gamma$. Figure-6 depicts a radio environment map constructed by the CRB based on the particle filter outputs corresponding to he ML estimates. In the figure we observe three (almost stationary) primary users being tracked by the CRB. The PU closer to the CRB is tracked with a low jitter in order to attain greater precision on its position.

\section{CONCLUSION}

A Bayesian tracking technique was presented to track primary users in a cognitive radio network to attain greater precision on their positional information. The tracking algorithm utilizes the fused positional information obtained by the individual cognitive radio nodes using maximum likelihood 


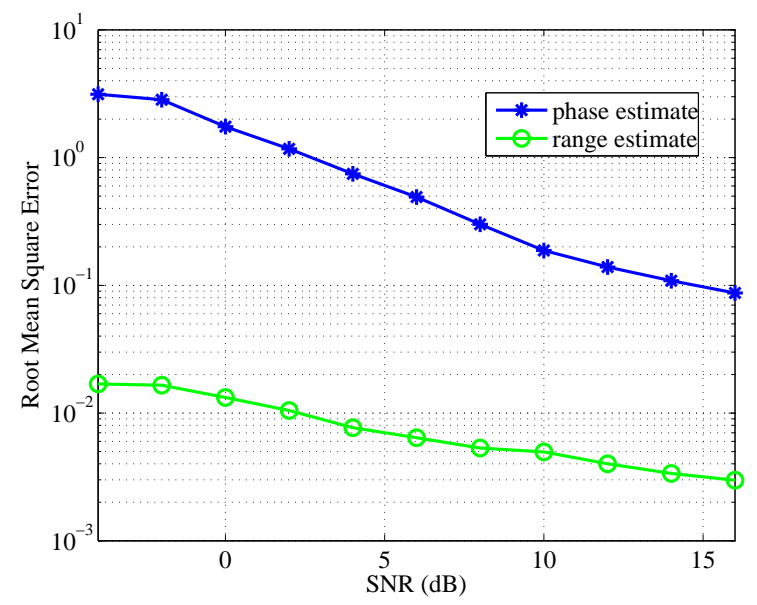

Fig. 5. Bayesian estimation root mean squared (rms) error for $d_{j}(k)$ and $\phi_{j}(k), M=100$

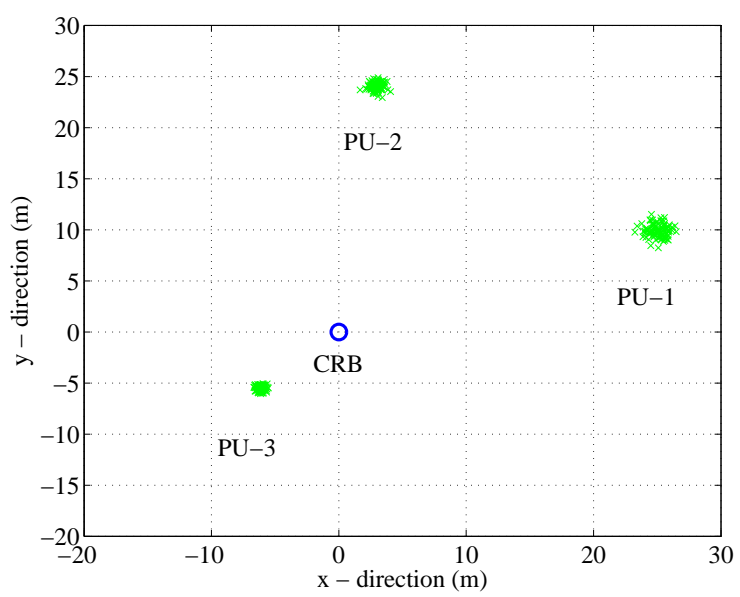

Fig. 6. Bayesian tracking of primary users; $d_{1}=26.9 \mathrm{~m}, \phi_{1}=21.8 \mathrm{deg}$, $d_{2}=24.2 \mathrm{~m}, \phi_{2}=82.9 \mathrm{deg}, d_{3}=8.2 \mathrm{~m}, \phi_{3}=222 \mathrm{deg}$

estimates. We present a tracking algorithm to construct a radio map environment which is suitable for tracking Gaussian and non-Gaussian noisy observations based on the posteriori density information. We present two unique importance-functions to reconstruct the posteriori distribution using the Bayesian technique. Simulation results are presented on the performances of the tracking algorithm, namely the convergence, the construction of the distribution, and the improvement in the jitter performances for a very slowly varying (almost static) system model.

\section{ACKNOWLEDGMENT}

The authors would like to thank the European Commission for partly funding this research under the Framework Program7 (EU-FP7) in the Information and Communications Technology cluster. EUWB (FP7-ICT-215669) [25].

\section{REFERENCES}

[1] J. Mitola and G. Maguire Jr., Cognitive Radio: Making Software Radios More Personal, IEEE Personal Communications, vol. 6, no. 4, pp. 1318, Aug. 1999.

[2] S. Haykin, Cognitive Radio: Brain-Empowered Wireless Communications, IEEE Journal on Select Areas in Commun., vol. 23, no. 2, pp. 201 - 220, Feb. 2005.
[3] H. Arslan (Ed), "Cognitive Radio, Software Defined Radio, and Adaptive Wireless Systems", Springer, Netherlands, 2007

[4] E. Hossain (Ed), V. Bhargava (Ed), 'Cognitive Wireless Communication Networks', Springer, New York, 2007

[5] Federal Communications Commission, Facilitating Opportunities for Flexible, Efficient, and Reliable Spectrum Use Employing Cognitive Radio Technologies, NPRM and Order, ET Docket no. 03-322, Dec. 2003.

[6] The Commission of the European Communities, Commission Decision 2007/131/EC on allowing the use of the radio spectrum for equipment using ultra-wideband technology in a harmonised manner in the Community, Official Journal of the European Union, Feb. 21, 2007.

[7] Brian D. O. A. and J. B. Moore, Optimal Filtering, Dower, Jan 2005

[8] Steven M. Kay, Fundamentals of Statistical Processing, Volume I: Estimation Theory, Prentice Hall, 1993

[9] Y. Oshman and P. Davidson, Optimization of observer trajectories bearingsonly target localization, IEEE Transactions on Aerospatial Electronical Systems, vol. 35, no. 3, pp. 892902, 1999.

[10] L. Kaplan, Q. Le, and P. Molnar, Maximum likelihood methods for bearingsonly target localization, in Proceedings of the 2001 IEEE ICASSP, Salt Lake City, TX, May 2001.

[11] S. Haykin, Array Signal Processing. Englewood Cliffs, NJ: Prentice Hall, 1984.

[12] K. Yao, R. Hudson, C. Reed, D. Chen, and F. Lorenzelli, Optimization of observer trajectories bearingsonly target localization, IEEE Jour on Selected Areas in Comms, vol. 16, no. 8, pp. 15551567, Oct. 1998.

[13] J. C. Chen, R.E. Hudson and K.Yao, A maximum likelihood parametric approach to source localization, IEEE ICASSP, Salt Lake City, TX, May 2001.

[14] R. Niu and P. K. Varshney, Target location estimation in sensor networks with quantized data, vol. 54, no. 12, pp. 45194528, Dec. 2006.

[15] D. Blatt and A. O. H. III, Energybased sensor network source localization via projection onto convex sets, vol. 54, no. 9, pp. 3614 3619, Sep. 2006.

[16] X. Sheng and Y. Hu, Maximumlikelihood multiplesource localization using acoustic energy measurements with wireless sensor networks, vol. 53, no. 1, pp. 4453, Jan. 2005.

[17] D. Li and Y. Hu, Energybased collaborative source localization using acoustic microsensor array, EURASIP Journal of Applied Signal Processing, no. 4, pp. 321337, 2003.

[18] M. G. Rabbat and R. D. Nowak, Decentralized source localization and tracking, in Proceedings of the 2004 IEEE conference ICASSP, Montreal, QC, Canada, 2004, pp. 921924.

[19] A. O. H. III and D. Blatt, Sensor network source localization via projection onto convex sets, in Proceedings of the 2005 IEEE International Conference on Acoustics, Speech, and Signal Processing, Philadelphia,PA, mar 2005.

[20] Randolph L. M., Dushyanth. K, Robert M. P.A Self-LocalizationMethod forWireless Sensor Networks, EURASIP Journal on Applied Signal Processing 2003:Vol.4,pp 348358

[21] M. S. Arulampalam, Simon Maskell, Neil Gordon, and Tim Clapp, A Tutorial on Particle Filters for Online Nonlinear/Non-Gaussian Bayesian Tracking, IEEE Trans on Signal Proc, Vol.50, No.2, Feb 2002

[22] P. M. Djuric et. al. "Particle Filtering", IEEE Signal Processing Magazine, September 2003.

[23] R. DI Girolamo and T. LE-NGOC, 'Frequency Independent Nonlinear Feedforward Phase Estimator', Wireless Personal Communications Journal, Kluwer Academic Publishers, Vol.5. No.1, July 1997, pp 19-50

[24] S. Kandeepan, Sam Reisenfeld, "Phase detector models and their performances for IF/baseband frequency recovery for complex envelope based DSP implemented PLL" IEEE conf ICCS 2002, 25-28 Nov, Singapore, pp:774-778

[25] URL of EUWB consortium http://www.euwb.eu 